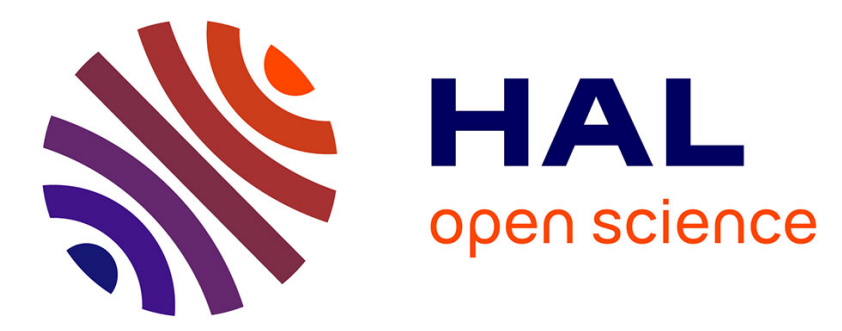

\title{
Estimation numérique du bruit rayonné par une turbulence homogène et isotrope, à l'aide d'un code de simulation directe et d'un code de simulation des grandes échelles
}

\author{
A. Witkowska, D. Juvé, J. Brasseur
}

\section{To cite this version:}

A. Witkowska, D. Juvé, J. Brasseur. Estimation numérique du bruit rayonné par une turbulence homogène et isotrope, à l'aide d'un code de simulation directe et d'un code de simulation des grandes échelles. Journal de Physique IV Proceedings, 1994, 04 (C5), pp.C5-939-C5-942. 10.1051/jp4:19945205 . jpa-00252889

HAL Id: jpa-00252889

https://hal.science/jpa-00252889

Submitted on 1 Jan 1994

HAL is a multi-disciplinary open access archive for the deposit and dissemination of scientific research documents, whether they are published or not. The documents may come from teaching and research institutions in France or abroad, or from public or private research centers.
L'archive ouverte pluridisciplinaire HAL, est destinée au dépôt et à la diffusion de documents scientifiques de niveau recherche, publiés ou non, émanant des établissements d'enseignement et de recherche français ou étrangers, des laboratoires publics ou privés. 


\title{
Estimation numérique du bruit rayonné par une turbulence homogène et isotrope, à l'aide d'un code de simulation directe et d'un code de simulation des grandes échelles
}

\section{A. WITKOWSKA, D. JUVÉ et J. BRASSEUR*}

Ecole Centrale de Lyon, Laboratoire de Mécanique des Fluides et d'Acoustique, URA 263 du CNRS, ECL-BP. 163, 69131 Ecully cedex, France

* Penn State University, Department of Mechanical Engineering, University Park, PA 16802, U.S.A.

\begin{abstract}
Lighthill's acoustic analogy is combined with a numerical simulation of homogeneous and isotropic turbulence in order to evaluate the farfield noise. Two different codes are used: a Large Eddy Simulation code and a Direct Numerical Simulation code. First, the form of the acoustic analogy to be used is analyzed. Then, the acoustic pressure is computed for several realizations of the turbulent field. Finally, nondimensional acoustic power is obtained from an ensemble average of acoustic pressure. The results computed with the two simulations are shown to be in good agreement.
\end{abstract}

\section{LA THEORIE DE LIGHTHILL}

En 1952 Lighthill [1] étudia le problème du rayonnement de bruit par un volume fini de turbulence situé dans un milieu au repos. Il exprima la pression acoustique rayonnée au point $\vec{x}$ en fonction des vitesses au sein du volume de turbulence V. L'expression exacte de la pression acoustique est:

$$
p(\vec{x}, t)=\frac{\rho_{0}}{4 \pi} \int_{V}\left[\frac{\partial^{2} u_{i} u_{j}}{\partial y_{i} \partial y_{j}}\right] \frac{1}{r} d V(\vec{y})
$$

ou, alternativement

$$
p(\vec{x}, t)=\frac{\rho_{0}}{4 \pi} \frac{\partial^{2}}{\partial x_{i} \partial x_{j}} \int_{V}\left[u_{i} u_{j}\right] \frac{1}{r} d V(\vec{y}) .
$$

où $c_{0}$ réprésente la vitesse du son, $\rho_{0}$ la densité du fluide, $\vec{r}=\vec{x}-\vec{y}$ et les termes entre parenthèses $[. .$.$] sont calculés au temps retardé t-\frac{r}{c_{0}}$. Avec l'hypothèse de champ lointain géométrique $(x \gg D)$ et de champ lointain acoustique $(x \gg \lambda)$, où $\mathrm{D}$ est une dimension caractéristique du volume $\mathrm{V}$ et $\lambda$ est la longueur d'onde du son émis, l'expression devient:

$$
p(\vec{x}, t)=\frac{\rho_{0}}{4 \pi c_{0}^{2}} \frac{x_{i} x_{j}}{x^{3}} \int_{V}\left[\frac{\partial^{2} u_{i} u_{j}}{\partial t^{2}}\right] d V(\vec{y}) .
$$

$\mathrm{Si}$, en outre, la condition de compacité $(\lambda \gg D)$ est satisfaite, on obtient:

$$
p(\vec{x}, t)=\frac{\rho_{0}}{4 \pi c_{0}^{2}} \frac{x_{i} x_{j}}{x^{3}}\left[\int_{V} \frac{\partial^{2} u_{i} u_{j}}{\partial t^{2}} d V(\vec{y})\right] .
$$

D'autre part, plusieurs auteurs ( Powell [2], Möhring [3], ...) proposèrent, par la suite, des formulations de la pression acoustique faisant intervenir la vorticité. 


\section{LES CODES DE SIMULATION}

Afin de connaître les vitesses au sein du volume $V$ nous avons utilisé deux codes de simulation de la turbulence: un code de simulation directe ( code DNS ) et un code de simulation des grandes échelles ( code LES ). Les deux codes simulent une décroissance de turbulence homogène et isotrope dans un cube avec des conditions de périodicité aux limites. Le nombre de Mach turbulent $M=u^{\prime} / c_{0}$, où u' est la vitesse efficace initiale est de 0.01 environ dans les deux cas.

Le code DNS a été élaboré par Ruetsch [4] et modifié par Wang [5] et nous l'avons utilisé avec un maillage de $64^{3}$. Le spectre initial est une combinaison d'une courbe en $k^{2}$ pour les petits nombres d'onde et d'une courbe en $k^{-5 / 3}$ pour les grands nombres d'onde. Le nombre de Reynolds initial, basé sur l'échelle de Taylor transversale est de 20 environ.

Quant au code LES, il a été developpé par Bertoglio [6]. Dans ce code, on tient compte des structures inférieures à la taille de la maille par l'intérmédiaire d'un modèle de sous-maille de CholetLesieur, ce qui parmet d'effectuer les calculs avec une résolution spatiale beaucoup plus faible. Nous avons donc utilisé un maillage de $16^{3}$ noeuds seulement. Le spectre initial est un spectre expérimental de turbulence de grille et le nombre de Reynolds initial est de 260 environ. Notons, par ailleurs, que nous avons obtenu des résultats similaires avec une résolution de $32^{3}$.

\section{COMBINAISON DE L'ANALOGIE DE LIGHTHILL ET DES CODES DE SIMU- LATION}

La combinaison de la théorie de Lighthill et du code de simulation pose de prime abord un problème essentiel. En effet, la théorie de Lighthill a été établie pour un domaine fini de turbulence situé dans un milieu au repos. Or, les deux codes numériques décrits plus haut, ne simulent qu'une partie tronquée du champ turbulent. Cette troncature peut être représentée formellement ( cf. [7] ) en multipliant le champ de vitesses par la fonction de Heavyside $\mathrm{H}(\mathrm{f})$, où $f(\vec{y})=0$ est l'équation de la surface limitant le volume de turbulence. La présence de la fonction $H(f)$ va introduire, dans toutes les formulations de la pression acoustique faisant intervenir des dérivées spatiales par rapport à $\vec{y}$, des termes supplémentaires non physiques sous forme d'intégrales de surface ( cf. [8] ). Ces termes, de nature dipolaire voire monopolaire risquent de l'emporter largement sur la contribution quadripolaire du domaine de turbulence. Par conséquent la formulation 1 ainsi que toutes les formulations faisant intervenir la vorticité sont à rejeter dans notre cas au profit des formulations 2,3 ou 4 .

\section{LES RESULTATS NUMERIQUES}

Nous avons donc calculé la pression acoustique à l'aide des deux codes de simulation et de la formulation 3, plus simple à employer que la formution 2. Le calcul a été fait en champ lointain. Le pas de temps a été choisi de manière à prendre en compte convenablement les temps retardés. Ainsi, pour le code LES nous avons choisi $\Delta t=\Delta d / c_{0}$, où d est la taille de la maille. Quant au code DNS, nous avons choisi un pas de temps de $2 \Delta d / c_{0}$ afin d'accelérer les calculs et après avoir vérifié, au préalable, que les résultats obtenus étaient identiques à ceux calculés avec un pas de temps de $\Delta d / c_{0}$. La puissance acoustique par unité de masse est $P=4 \pi I /\left(\rho_{0} V\right)$, ò̀ I est l'intensité acoustique (cf. $[9]):$

$$
I=\frac{\left\langle(p-<p>)^{2}>\right.}{\rho_{0} c_{0}} .
$$

Les crochets désignent ici les moyennes statistiques, que nous avons calculées sur une cinquantaine de réalisations pour le code LES et sur une dizaine de réalisations pour le code DNS.

Nous avons rendu adimensionelle la puissance acoustique en la divisant par $u^{\prime 3} / l$ et le temps en le divisant par $l / u^{\prime}$, où u' est la vitesse efficace initiale et 1 l'échelle intégrale initiale de la turbulence. Comme les nombres de Mach sont égaux on peut alors comparer directement les résultats obtenus à l'aide des deux codes. 
Enfin, nous avons comparé nos résultats au modèle de Proudman. En effet, Proudman [9] parvient à exprimer la puissance acoustique rayonnée par unité de masse d'une turbulence homogène et isotrope sous la forme $P=\alpha M^{5} \epsilon$, avec $\mathrm{M}$ le nombre de Mach turbulent, $\epsilon$ la dissipation et $\alpha$ un coefficient qui dépend du spectre de la turbulence et vaut 38 pour le spectre de Heisenberg. M et $\epsilon$ peuvent être calculés à l'aide des deux codes.

Sur la figure 1 nous avons donc représenté, d'une part, la puissance acoustique calculée à l'aide de la formulation 3 et des codes LES et DNS, et, d'autre part, la puissance acoustique obtenue avec le modèle de Proudman et les deux codes. Il apparaît, tout d'abord, que les résultats obtenus avec les deux codes concordent bien, si ce n'est une legère différence de la vitesse de décroissance de la puissance acoustique. Elle est sans doute due a une petite différence entre les lois de décroissance de l'énergie turbulente données par les deux codes. D'autre part, on observe que la puissance acoustique obtenue à l'aide de l'analogie de Lighthill, est inférieure de $10 \mathrm{~dB}$ environ aux prévisions de Proudman. Cette différence peut avoir plusieurs origines. Tout d'abord la valeur $\alpha=38$ est spécifique du spectre de Heisenberg. Or, les spectres des deux codes numériques sont différents du spectre de Heisenberg. Par exemple, la valeur de $\alpha$ calculée pour le spectre de Von Karman tronqué du code LES se revèle voisine de 10. D'autre part, pour trouver l'expression de $\alpha$, Proudman utilise l'hypothèse de quasinormalité, très discutable.

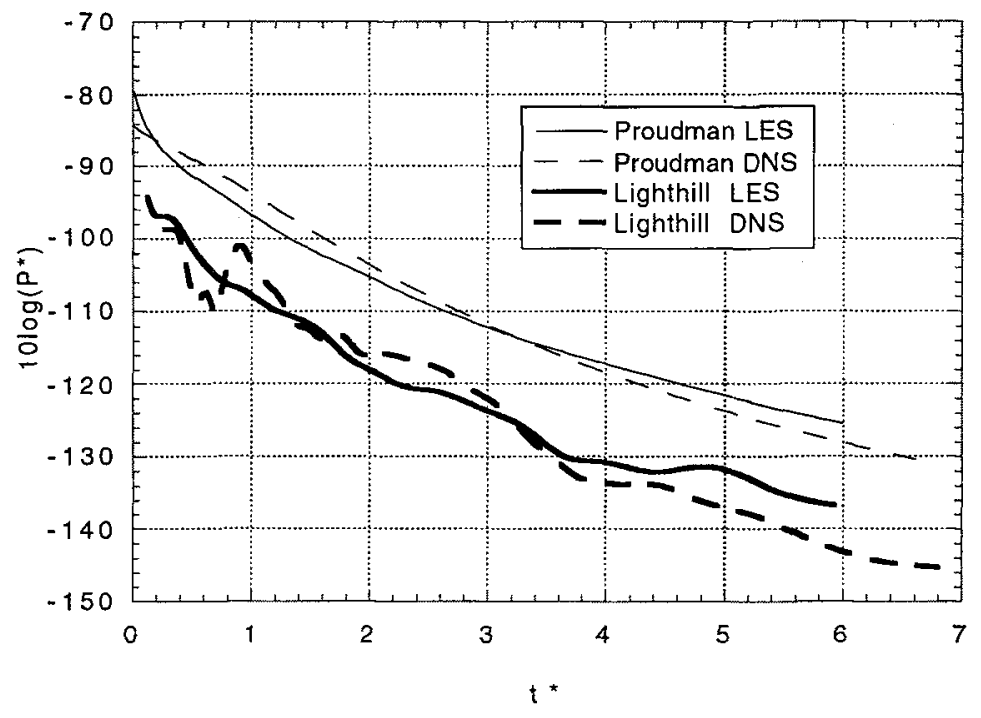

Figure 1: Évolution au cours du temps de la puissance acoustique rayonnée par unité de masse avec

$$
P^{*}=\frac{P}{u^{\prime 3} / l} \quad \text { et } \quad t^{*}=\frac{t}{l / u^{\prime}}
$$

\section{CONCLUSION}

La combinaison de la théorie de Lighthill avec deux codes de simulation différents permet donc d'obtenir des puissances acoustiques par unité de masse sensiblement égales. De plus, nos résultats concordent avec ceux de Sarkar [10], qui utilise un code DNS, des moyennes temporelles et la formulation 3 et obtient une puissance acoustique inférieure de $10 \mathrm{~dB}$ environ aux prévisions de Proudman. 
Cette concordance entre les différents résultats nous paraît encourageante. Notons, cependant, qu'elle n'est obtenue qu'à condition d'utiliser une formulation appropriée de la pression acoustique. En effet, Béchara [11], qui emploie de façon erronée la formulation 1 obtient une puissance acoustique supérieure de $40 \mathrm{~dB}$ à nos résultats. Cette large surestimation est due aux termes monopolaires et dipolaires présents dans les intégrales de surface contenues implicitement dans cette formulation.

\section{Références}

[1] Lighthill M.J., Proc.Roy.Soc.London, Vol 1 (1952) pp. 564-587.

[2] Powell A., J.Acoust.Soc.America, Vol 36 (1964) pp. 177-195.

[3] Möhring W., J.Fluid.Mech., Vol 85, part 4 (1978) pp. 685-691.

[4] Reutsch G.R. \& Maxey M.R., Phys. Fluids A Vol 3 (1991) pp. 1587-1597.

[5] Wang L.-P. and Maxey M.R., J.Fluid Mech., Vol 256 (1993) pp. 27-68.

[6] Bertoglio J.P., Etude d'une turbulence anisotrope; modélisation de sous-maille et approche statistique, Thèse de Doctorat d'Etat es Sciences, Université Claude Bernard, Lyon I, (1986).

[7] Crighton D.J., Dowling A.P., Ffowcs Williams J.E., Heckl M. \& Leppington F.G., Modern Methods in Analytical Acoustics (Springer-Verlag, 1992) pp. 46-79.

[8] Witkowska A.\& Juvé D., "Simulation numérique du bruit crée par une turbulence homogène et isotrope", treizième Colloque d'Aéro et Hydroacoustique, Ecole Centrale de Lyon France juin 1993.

[9] Proudman I., Proc.Roy.Soc., A 214 (1952) pp. 119-132.

[10] Sarkar S.\& Hussaini M.Y., Computational aeroacoustics (eds Hardin \& Hussaini, Springer Verlag, 1993) pp. 335-351.

[11] Béchara W., Modélisation du bruit d'écoulements turbulents libres, Thèse de Doctorat, Ecole Centrale de Paris, (1992). 